\title{
Protease-Activated Receptors 1 and 3 are Differentially Expressed on Human Monocyte Subsets and are Upregulated by Lipopolysaccharide Ex Vivo and In Vivo
}

\author{
${ }^{1}$ Department of Internal Medicine II, Medical University of Vienna, \\ Vienna, Austria \\ 2 Department of Clinical Pharmacology, Medical University of Vienna, \\ Vienna, Austria \\ ${ }^{3}$ Department of Internal Medicine I, Medical University of Vienna, \\ Vienna, Austria \\ ${ }^{4}$ Clinic for Blood Group Serology and Transfusion Medicine, Medical \\ University of Vienna, Vienna, Austria \\ ${ }^{5}$ Department for Health Science and Biomedicine, Danube University \\ Krems, Krems, Austria \\ 6 3rd Medical Department for Cardiology and Emergency Medicine, \\ Wilhelminen Hospital, Vienna, Austria \\ ${ }^{7}$ Ludwig Boltzmann Cluster for Cardiovascular Research, Vienna, \\ Austria \\ ${ }^{8}$ Core Facilities, Medical University of Vienna, Vienna, Austria
}

Barbara Thaler ${ }^{1}$ Philipp J. Hohensinner ${ }^{1}$ Johanna Baumgartner ${ }^{1}$ Patrick Haider ${ }^{1}$ Konstantin A. Krychtiuk ${ }^{1}$ Christian Schörgenhofer ${ }^{2}$ Bernd jilma ${ }^{2}$ Lena Hell ${ }^{3}$ Michael B. Fischer ${ }^{4,5}$ Kurt Huber $^{6}$ Christian Hengstenberg ${ }^{1}$ Walter S. Speidl ${ }^{1}$ Johann Wojta ${ }^{1,7,8}$ Address for correspondence Johann Wojta, PhD, Department of Internal Medicine II, Medical University of Vienna, Waehringer Guertel 18-20,

Thromb Haemost 2019;119:1394-1402.

\begin{abstract}
Keywords

- monocyte subsets

- protease-activated receptors

- low-grade inflammation

- coagulation
\end{abstract}

Monocytes are activated in inflammatory conditions via a variety of cytokine receptors as well as in a procoagulatory setting through thrombin, acting upon protease-activated receptors (PARs). This study investigated the expression pattern of PAR1 and PAR3 on human monocyte subsets. Furthermore, a possible regulation of the expression of PAR1 and PAR3 in these cells by inflammatory activation were studied. CD16 $6^{+}$monocytes showed significantly higher levels of PAR1 and PAR3 as compared with $\mathrm{CD} 16^{-}$monocytes. Ex vivo treatment of whole blood with lipopolysaccharide (LPS) increased PAR1 and PAR3 messenger ribonucleic acid (mRNA) in human monocytes. In addition, increase of PAR1 was seen in all three subsets upon LPS treatment, whereas PAR3 increased significantly only in $\mathrm{CD}_{16}{ }^{-}$monocytes and nonclassical CD16 ${ }^{+}$monocytes. Protein levels of PAR1 and PAR3 significantly increased on monocytes in vivo in human endotoxemia 1 hour after LPS infusion. PAR1 increased significantly in $\mathrm{CD} 16^{-}$monocytes and nonclassical CD16 ${ }^{+}$ monocytes. In this in vivo model, PAR3 was also significantly elevated in $\mathrm{CD}_{16}^{-}$monocytes and increased slightly albeit not significantly in $\mathrm{CD}_{16}{ }^{+}$monocytes. Endotoxemia increased plasminogen activator inhibitor-1 (PAI-1) and tissue factor (TF) expression in monocytes in humans. Pretreatment of healthy volunteers with the PAR1 antagonist vorapaxar blocked the increase in PAI-1 but not the increase in TF. We here provide new evidence for a critical role for monocytes as cellular mediators that contribute to the activation of coagulation in diseases characterized by an inflammatory state. received

November 17, 2018

accepted after revision

April 29, 2019
DOI https://doi.org/

$10.1055 / \mathrm{s}-0039-1692219$.

ISSN 0340-6245. (c) 2019 Georg Thieme Verlag KG Stuttgart · New York
License terms

(요 $\Theta \circledast$ 


\section{Introduction}

Monocytes are cellular mediators linking inflammation and coagulation. On the one hand, they express a variety of specific cytokine receptors which render them responsive to activation by a host of inflammatory mediators. ${ }^{1}$ On the other hand, monocytes express protease-activated receptors (PARs). ${ }^{2}$ PARs are $G$ protein-coupled transmembrane receptors that are activated through proteolytic cleavage of their tethered ligand. ${ }^{3}$ Currently, four PARs denominated PAR1, 2, 3, and 4 have been identified. ${ }^{4}$ The serine protease and central component of the coagulation cascade, thrombin, can cleave all PARs whereby PAR2 is only activated by high concentrations of thrombin. ${ }^{4}$ In addition to thrombin, other proteases involved in thrombus formation and dissolution such as the coagulations factors $\mathrm{Xa}$ and VIIa, activated protein C, and plasmin have been shown to activate PARs. Furthermore, besides monocytes other cells regulating vascular integrity and the vasculature's response to injury and inflammatory activation, such as endothelial cells and smooth muscle cells, express PARs. ${ }^{5,6}$ Monocytes have been shown to express mainly PAR1 and PAR3. ${ }^{7}$ Thus, monocytes through the interaction of thrombin with PAR1 and PAR3 can also be activated in a procoagulatory environment.

Human monocytes, however, are not a homogeneous cell population. Based on their expression of the surface markers CD14 and CD16, three subsets can be distinguished: CD14 ${ }^{++} \mathrm{CD} 16^{-}$classical monocytes (Mon1), CD14 ${ }^{++} \mathrm{CD} 16^{+}$ intermediate monocytes (Mon2), and $\mathrm{CD} 14^{+} \mathrm{CD} 16^{++}$nonclassical monocytes (Mon3). ${ }^{8,9}$ In various clinical studies an association of $\mathrm{CD} 16^{+}$subsets with pathologies characterized by a chronic inflammatory state such as coronary artery disease, obesity, arthritis, inflammatory diseases of the intestinal tract, or systemic lupus erythematosus has been described. ${ }^{10-14}$ Recently, we showed that Mon2 were associated with 30-day mortality in critically ill patients. ${ }^{15}$ Furthermore, we established in a human endotoxemia model that especially $\mathrm{CD} 16^{+}$cells respond to lipopolysaccharide (LPS)-induced activation with an increased production of the inflammatory cytokines interleukin-6 (IL-6) and IL-8. ${ }^{16}$

However, the expression of PAR1 and PAR3 on the three subsets of human monocytes has not been studied yet and information on possible regulation of these receptors under inflammatory conditions is scarce. ${ }^{7}$ Therefore, we investigated if human monocyte subsets differentially express PAR1 and PAR3. Furthermore, we investigated ex vivo and in vivo if the expression of PAR1 and PAR3 by human monocytes is affected by inflammatory activation.

Finally, we performed a randomized, placebo-controlled clinical trial to study the influence of the PAR1-antagonist vorapaxar on plasminogen activator inhibitor-1 (PAI-1) and tissue factor (TF) expression during human endotoxemia, a well standardized model of inflammation-induced coagulation. ${ }^{17,18}$

\section{Materials and Methods}

\section{Immunofluorescence Microscopy}

Peripheral blood mononuclear cells (PBMCs) were isolated from whole blood samples and were seeded into chamber slides. Monocytes were left to adhere for 3 hours at $37^{\circ} \mathrm{C}$ and afterwards non attached cells were washed off with phosphate-buffered saline (PBS), $\mathrm{pH}=7.4$. Subsequently, monocytes were fixed with $4 \%$ paraformaldehyde, permeabilized with $0.1 \%$ triton $\mathrm{X}$, and stained with primary antibodies against PAR1 (Thermo Fisher Scientific, Waltham, United States, host mouse, dilution 1:100) and PAR3 (Santa Cruz Biotechnology, Santa Cruz, California, United States, host rabbit, dilution 1:50). Cells were incubated over night with secondary antibodies (Cy3-anti-rabbit, BioLegend, San Diego, California, United States, and DyLight650-anti-mouse, Thermo Fisher Scientific, both with a dilution of $1: 200$ ), washed with PBS, and mounted with ProLong Gold antifade reagent with 4',6-diamidino-2-phenylindole (Thermo Fisher Scientific). Slides were analyzed on an LSM 700 confocal microscope with a $63 \times$ objective (Zeiss, Oberkochen, Germany). Magnification was further increased using the digital zoom option in the ZEN 2012 black software (Zeiss).

\section{Flow Cytometry}

For analysis of PAR1 and PAR3 levels in vivo, whole blood samples of healthy controls were stained with anti-CD 45 (Clone H130, APC/Cy7), anti-CD14 (Clone HCD14, PerCP), antiCD16 (Clone 3G8, PE, all BioLegend), and the respective antiPAR1 (clone ATAP2, fluorescein isothiocyanate [FITC]) or antiPAR3 (clone H-103) antibodies (Santa Cruz). The anti-PAR3 antibody was labeled with a FITC conjugation kit (Abcam). Samples were processed as previously published ${ }^{16}$ and measured on a BD Canto II flow cytometer. Note that 100,000 events were recorded and analyzed with the BD Diva 6 software. To investigate if inflammatory activation affects PAR levels on the respective cell surface, whole blood samples were incubated ex vivo with LPS ( $1 \mu \mathrm{g} / \mathrm{mL}$, Sigma Aldrich, Missouri, United States) for 4 hours at $37^{\circ} \mathrm{C}$ and afterwards cells were stained and analyzed as described above. In addition, whole blood samples were incubated with $1 \mu \mathrm{L} / \mathrm{mL}$ GolgiPlug reagent (BD Biosciences, San Jose, California, United States) as recommended by the manufacturer for 30 minutes at $37^{\circ} \mathrm{C}$ prior to LPS challenge to block transport of newly produced proteins from the Golgi apparatus by brefeldin A. After the stimulation with LPS for 4 hours, blood samples were processed for flow cytometric analysis as described above. Gating was performed as published recently. ${ }^{16}$

\section{Isolation and Stimulation of Monocytes}

PBMCs were isolated via density gradient centrifugation using lymphocyte separation medium 1077 (PromoCell, Heidelberg, Germany) from leukapheresis chambers obtained from healthy thrombocyte donors according to the recommendations of the ethical board of the Medical University of Vienna including informed consent. Afterwards, PBMCs were isolated using a monocyte negative selection kit (StemCell, Vancouver, Canada). Purity was tested with a Sysmex XM-350 (Kobe, Japan) and preparations with purity $>95 \%$ were used for further experiments.

Isolated monocytes were cultured in RPMI 1640 containing XerumFree (TNCBIO, Eindhoven, Netherlands) and 1\% penicillin/ streptomycin (Lonza, Basel, Switzerland) in u-bottom-shaped 
wells (Greiner, Kremsmuenster, Austria) and were stimulated for 6 hours with LPS $(1 \mu \mathrm{g} / \mathrm{mL}$, Sigma) or with thrombin receptor-activating peptide (TRAP)-6 (100 $\mu \mathrm{M}$, bio-techne, Minneapolis, Minnesota, United States). Afterwards, cells were harvested and RNA was isolated using a Maxwell 16 instrument and the respective simplyRNA kit (Promega, Madison, Wisconsin, United States).

\section{Assay for Tissue Factor Activity on Microvesicles}

Monocytes were isolated, seeded, and stimulated as described above. After the incubation time of 6 hours, monocytes were harvested, centrifuged, and the supernatants were collected for isolation of microvesicles (MVs) as described recently. ${ }^{19,20}$ Therefore, MVs were centrifuged at 14,000 revolutions per minute for 15 minutes at $4^{\circ} \mathrm{C}$ and washed twice with Hank's Balanced Salt Solution containing $0.1 \%$ bovine serum albumin (HBSA). Afterwards, MVs were incubated with either mouse anti-human TF antibody $(1 \mu \mathrm{g} /$ $\mathrm{mL}$, BD Biosciences) or a mouse immunoglobulin $\mathrm{G}$ control antibody ( $1 \mu \mathrm{g} / \mathrm{mL}$, Sigma) for 15 minutes at room temperature. Then, HBSA containing $10 \mathrm{mM} \mathrm{CaCl} 2$ together with factor X (300 nM, Coachrom, Maria Enzersdorf, Austria) and VIIa (10 nM, Coachrom) was added to the samples. After an incubation time of 2 hours, Pefachrome FXa 8595 (4 nM, Pentapharma, Geneva, Switzerland) was added and incubated at $37^{\circ} \mathrm{C}$ for 15 minutes. Afterwards, absorbance was measured at $405 \mathrm{~nm}$.

\section{Endotoxemia Model}

Healthy volunteers $(n=16)$ received either a dose of the PAR1 antagonist vorapaxar (10 mg, Merck, Darmstadt, Germany) or placebo. Twenty-four hours thereafter all participants received a bolus infusion of LPS (2 ng/kg; Clinical Center Reference Endotoxin lot from National Institutes of Health). ${ }^{21}$ Blood samples were taken 24 hours before infusion and 1 hour after LPS administration. Monocytes were isolated as described above and were used for RNA isolation. Study participants were apparently healthy with no symptoms of relevant clinical illness or medication. In addition, no blood donation was allowed within the last 4 weeks before inclusion. Furthermore, volunteers had no liver or kidney dysfunction and passed a physical examination with normal status for general appearance, head, ears, throat, nose, lymph nodes, cardiovascular, respiratory, abdomen, musculoskeletal, and neurologic status. This study was approved by the ethics committee of the Medical University of Vienna and performed according to the Declaration of Helsinki. Written and oral informed consent to participate in the study was obtained from all healthy volunteers before any studyrelated activity was performed.

\section{Real-Time Polymerase Chain Reaction}

Quantitative polymerase chain reactions (qPCRs) were performed with a Light Cycler 480 II (Roche Molecular Diagnostics, Basel, Switzerland) using the universal probe library system (UPL). Primers were designed with the online UPL tool (Roche). The PAR-1 primers had the sequences "tcagaagatgcctccggata" and "cacagatgggacaaagagtgtc" with UPL \#17 and the PAR3 primers "ctacgtccaggccacctcta" and "gtgaagtggtggagggtagg" with UPL \#86.

\section{Statistics}

Data are shown as mean \pm standard deviation. Samples were compared using Wilcoxon rank sum test or Mann-Whitney test. Calculations were performed using SPSS21, $p<0.05$ was considered statistically significant.

\section{Results}

As can be seen from the first row of images of - Fig. 1A, human monocytes express PAR1 and PAR3 as determined by immunohistochemistry. A combination of the primary rabbit anti-PAR3 antibody with the secondary DyLight650-antimouse antibody and a combination of the primary mouse anti-PAR1 antibody with the secondary Cy3-anti-rabbit antibody were used to control for species cross-reaction. No such cross-reaction was seen as depicted in the second and third row of images of - Fig. 1A, respectively. The gating strategy for human monocyte subsets in whole blood is depicted in -Fig. 1B. When human monocyte subsets in whole blood were analyzed by flow cytometry, CD16 ${ }^{+}$Mon2 and Mon3 showed significantly higher levels of PAR1 and PAR3 as compared with CD16 ${ }^{-}$Mon1 (-Fig. 1C and D).

When human whole blood was treated ex vivo with LPS, a significant increase in PAR1 and PAR3 protein on monocytes was observed by flow cytometry as compared with monocytes from whole blood not treated with LPS. To investigate whether the increased levels of PARs on the surface of these cells in response to LPS originate from an intracellular storage pool, GolgiPlug, which blocks the transport of protein from the endoplasmic reticulum to the Golgi apparatus, was used. The GolgiPlug did not significantly affect the LPSinduced increase of neither PAR1 nor PAR3 on the surface of monocytes (-Fig. 2A and B). As determined by real-time PCR, no significant changes in PAR1 and PAR3 mRNA levels in monocytes from LPS-treated whole blood as compared with mRNA levels in monocytes from untreated whole blood were observed (PAR1 under LPS treatment $3.9 \pm 3.3$ fold vs. control, $p=0.068$; PAR3 under LPS treatment $10.8 \pm 8.3$ fold vs. control, $p=0.068$; data not shown). When PAR1 and PAR3 levels were analyzed by flow cytometry on monocyte subsets from whole blood treated with LPS and compared with the respective levels on monocyte subsets from untreated blood, a significant increase in PAR1 was seen in all three subsets. PAR3 increased significantly only in Mon1 and Mon3, whereas the increase seen in Mon2 did not reach significance (-Fig. 2C and D).

Endotoxemia significantly increased PAR1 and PAR3 protein on monocytes 1 hour after LPS bolus as compared with monocytes collected from these individuals before LPS infusion ( - Fig. 3A and B). Furthermore, a similar pattern to that in the ex vivo situation was observed when PAR1 and PAR3 levels were determined on monocyte subsets before and 1 hour after LPS infusion in these volunteers. PAR1 increased significantly in Mon1 and Mon3. However, in contrast to our ex vivo experiments no significant change was seen in PAR1 
DyLight650
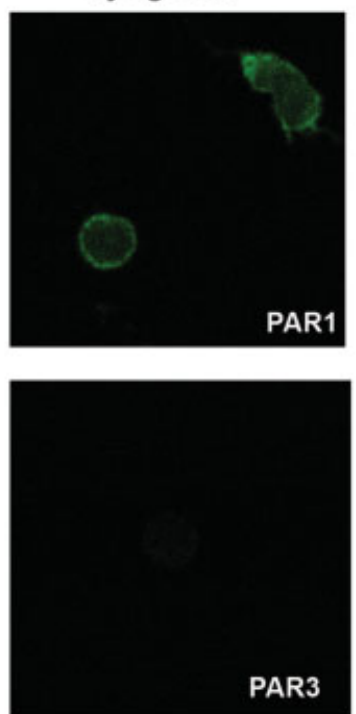

Cy3

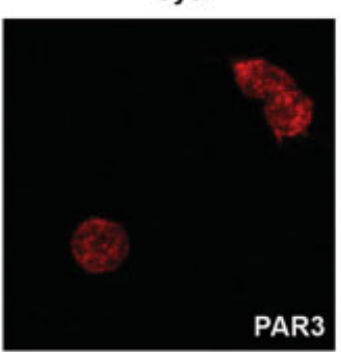

PAR3
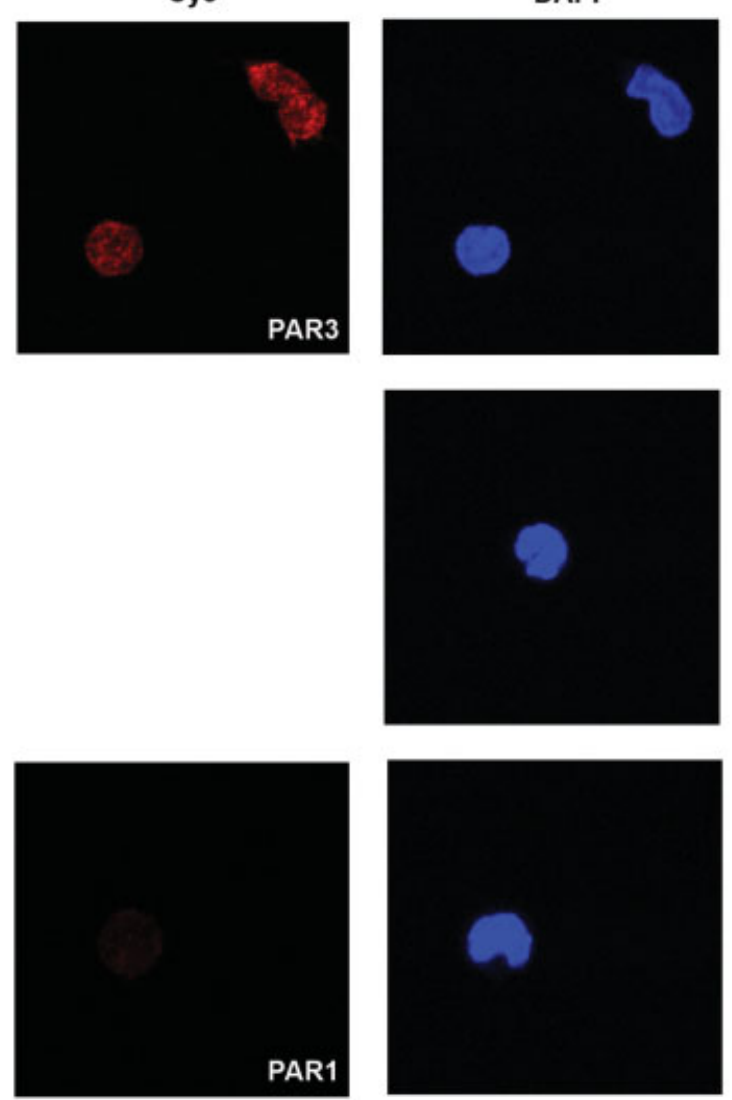

merged
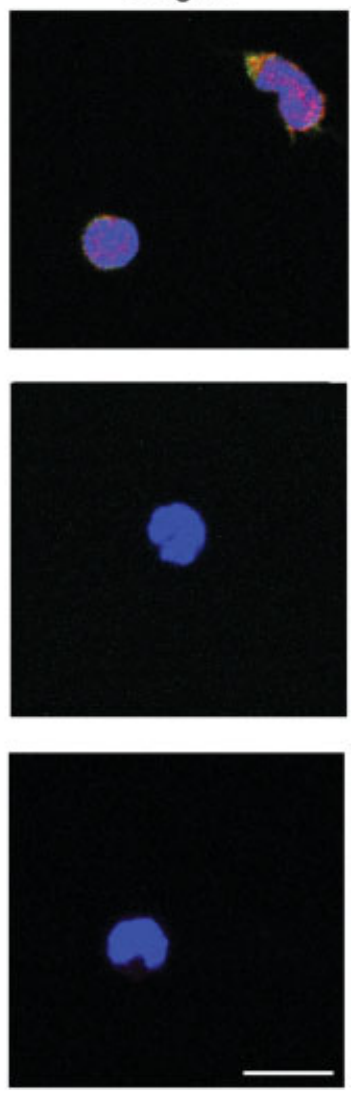

A
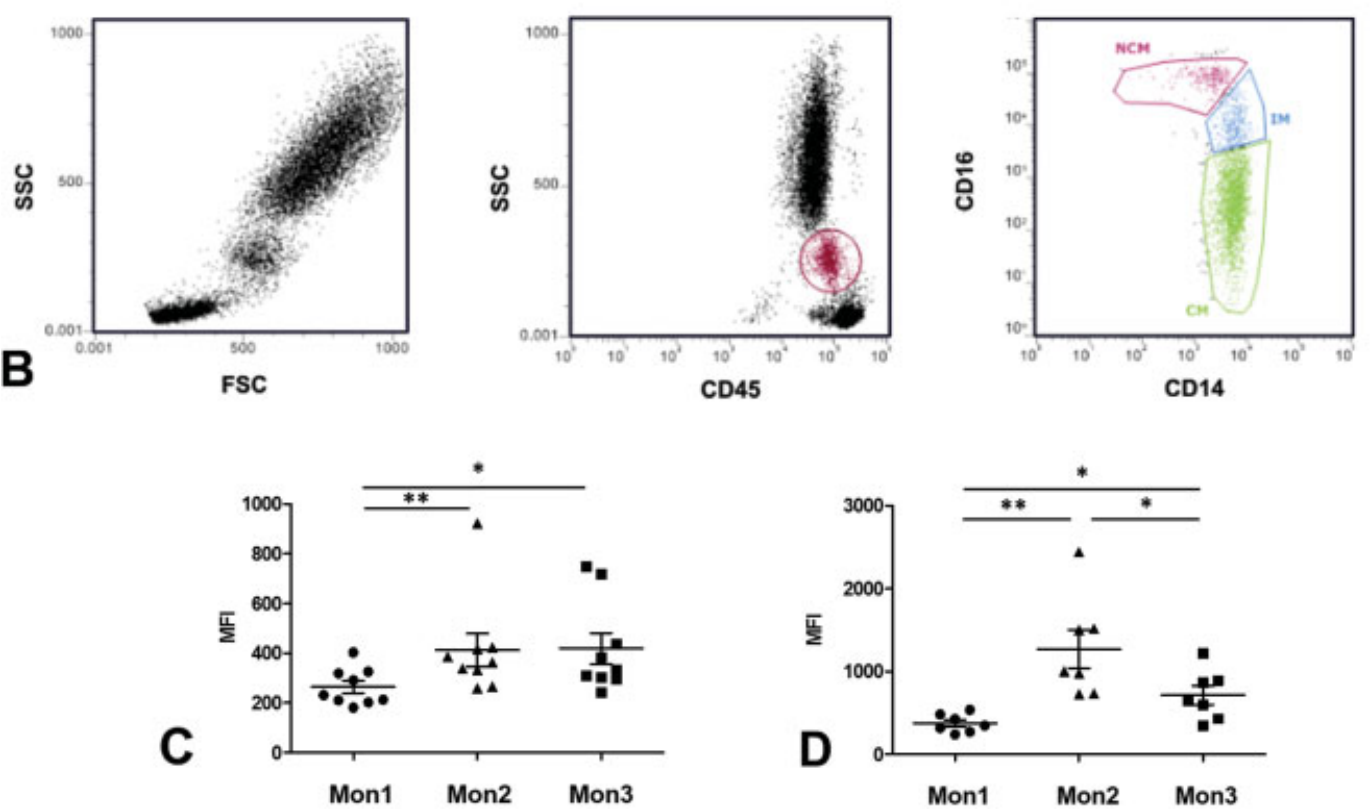

Fig. 1 Protease-activated receptor (PAR) 1 and PAR3 on human monocyte subsets. Immunohistochemistry staining of PAR1 (green) and PAR3 (red) and staining of nuclei with 4',6-diamidino-2-phenylindole (DAPI) (blue) was performed on monocytes isolated from three apparently healthy volunteers as described under the "Materials and Methods" section. A representative image of monocytes from one donor stained with the primary mouse anti-PAR1 antibody and the secondary DyLight650-anti-mouse antibody and with the primary rabbit anti-PAR3 antibody and the secondary Cy3-anti-rabbit antibody, respectively, is shown in the first row of images of panel A. Staining with a combination of the primary rabbit anti-PAR3 antibody with the secondary DyLight650-anti-mouse antibody and with a combination of the primary mouse anti-PAR1 antibody with the secondary Cy3-anti-rabbit antibody are shown in the second and third row of images of panel A, respectively. A size bar representing 10 $\mu \mathrm{M}$ is shown in white. Gating strategy of human monocyte subsets in whole blood (B). Flow cytometric analysis of levels of PAR1 (C; $n=9)$ and PAR3 ( $; ; n=7$ ) on monocyte subsets isolated from apparently healthy donors was performed as described in detail under the "Materials and Methods" section. Values are given as mean fluorescent intensities (MFIs) \pm standard deviation (SD); ${ }^{*} p<0.05,{ }^{* *} p<0.01$. 

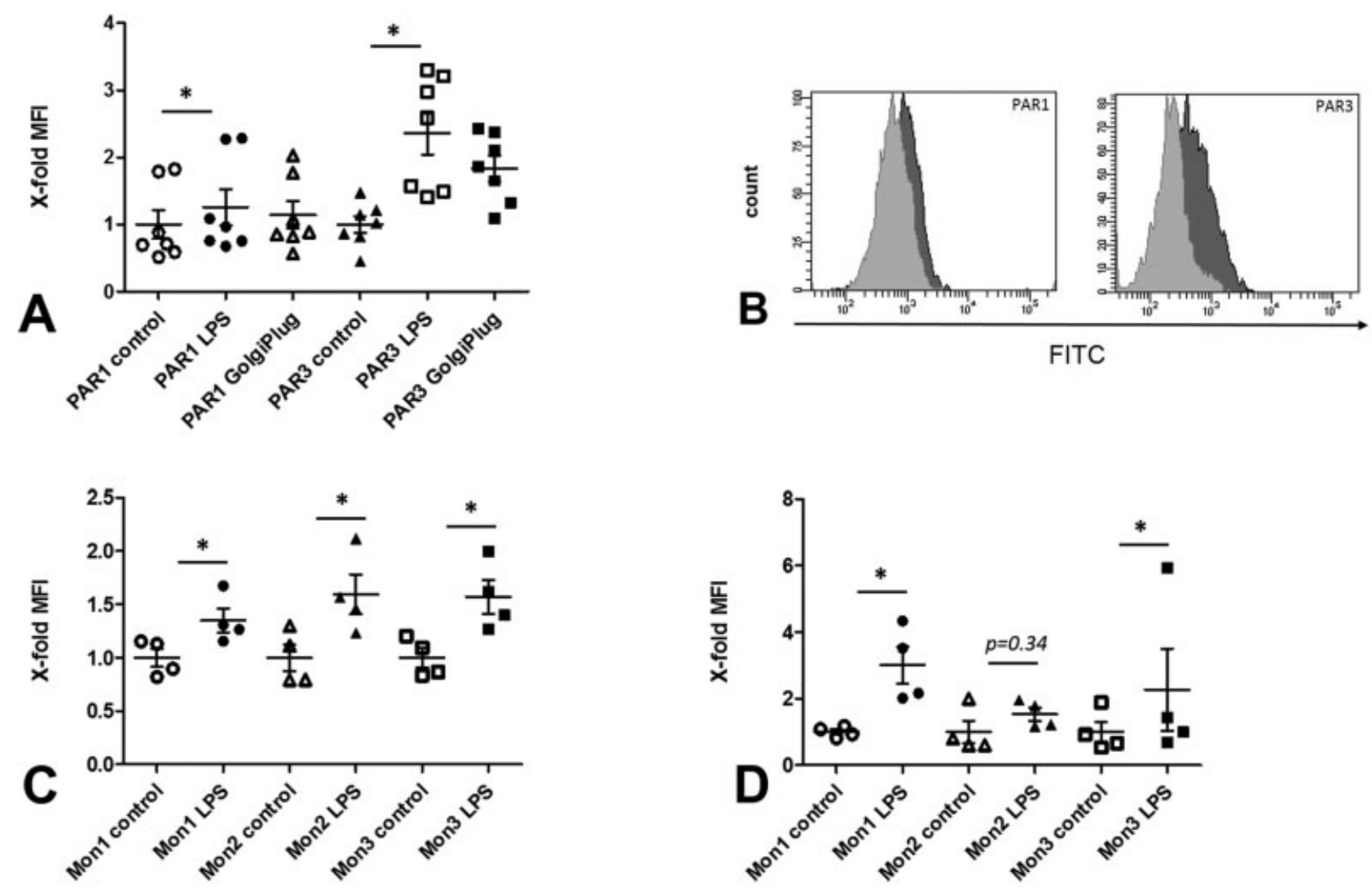

Fig. 2 Induction of protease-activated receptor (PAR) 1 and PAR3 on monocyte subsets by lipopolysaccharide (LPS) ex vivo. (A) Flow cytometric analysis of protein levels of PAR 1 and PAR3 on total monocytes from whole blood from apparently healthy volunteers that was treated for 4 hours at $37^{\circ} \mathrm{C}$ with $1 \mu \mathrm{g} / \mathrm{mL}$ LPS in the presence or absence of $1 \mu \mathrm{L} / \mathrm{mL}$ GolgiPlug reagent versus an untreated control $(n=7)$. (B) Representative histograms of monocytes stained for PAR1 and PAR3 from untreated blood samples or from blood samples treated with LPS from one donor. (C and D) PAR1 and PAR3 protein levels, respectively, as determined by flow cytometry on monocyte subsets from blood samples from apparently healthy volunteers treated 4 hours at $37^{\circ} \mathrm{C}$ with $1 \mu \mathrm{g} / \mathrm{mL}$ LPS or left untreated $(n=4)$. Values are displayed as $x$-fold mean fluorescent intensities (MFIs) versus unstimulated control \pm standard deviation (SD) (A, C, and D); ${ }^{*} p<0.05$.

in Mon2. In agreement with the ex vivo model, in the in vivo situation PAR3 was significantly elevated in Mon1 and increased slightly albeit not significantly in Mon2, whereas in contrast to the ex vivo setting the increase in PAR3 in Mon3 did not reach significance ( - Fig. 3 C and $\mathbf{D}$ ).

Thereafter, we tested whether LPS would affect a PARmediated response in monocytes in vivo. In the human endotoxemia model, volunteers $(n=16)$ received the PAR1 antagonist vorapaxar 24 hours prior to LPS infusion (10 mg, orally) or placebo before LPS infusion in a randomized crossover design, and the alternative treatment after a minimum washout period of 6 weeks.

Specific mRNAs for two proteins critically involved in thrombus formation and stabilization, namely, TF and PAI-1, were tested in monocytes obtained from these volunteers. 22,23 Endotoxemia significantly increased mRNAs specific for PAI-1 by approximately fourfold and TF by approximately ninefold in monocytes obtained from volunteers receiving LPS and placebo ( - Fig. 4A). Pretreatment with vorapaxar prior to LPS infusion nearly abolished the increase in PAI-1-specific mRNA, whereas the increase in TF-specific mRNA was not affected. It should be noted that it has been shown that LPS increases the expression of TF in monocytes whereas no such effect was reported for PAI- $1 .{ }^{24-28}$ In accordance with these reports, in monocytes treated with LPS in vitro PAI-1 expression was not affected, whereas TF expression and activity increased significantly in response to LPS. However, stimulation with the PAR-1 agonist TRAP-6 induced a significant increase in PAI-1 but did neither affect TF mRNA expression in total monocytes isolated from whole blood of apparently healthy volunteers nor TF activity on MVs shed by these cells ( - Fig. $\mathbf{4 B}$ and $\mathbf{C}$ ).

\section{Discussion}

Monocytes represent a cellular link between the immune and the coagulation system as they can not only be activated in an inflammatory environment by different cytokines acting upon a variety of different cytokine receptors but also in a procoagulatory setting by thrombin, the central protease of the coagulation cascade, through PARs. This link is operative, for example, in sepsis when monocytes react with increased production of TF, which in turn results in the generation of thrombin that acts upon PARs. ${ }^{29,30}$

However, monocytes are not a homogeneous cell population. Three subsets of monocytes have been identified in human blood based on their differential expression of the surface markers CD14 and CD16. ${ }^{8,9}$ It has been shown that human monocytes express PAR1, 2 , and $3 .{ }^{7}$ However, at least 

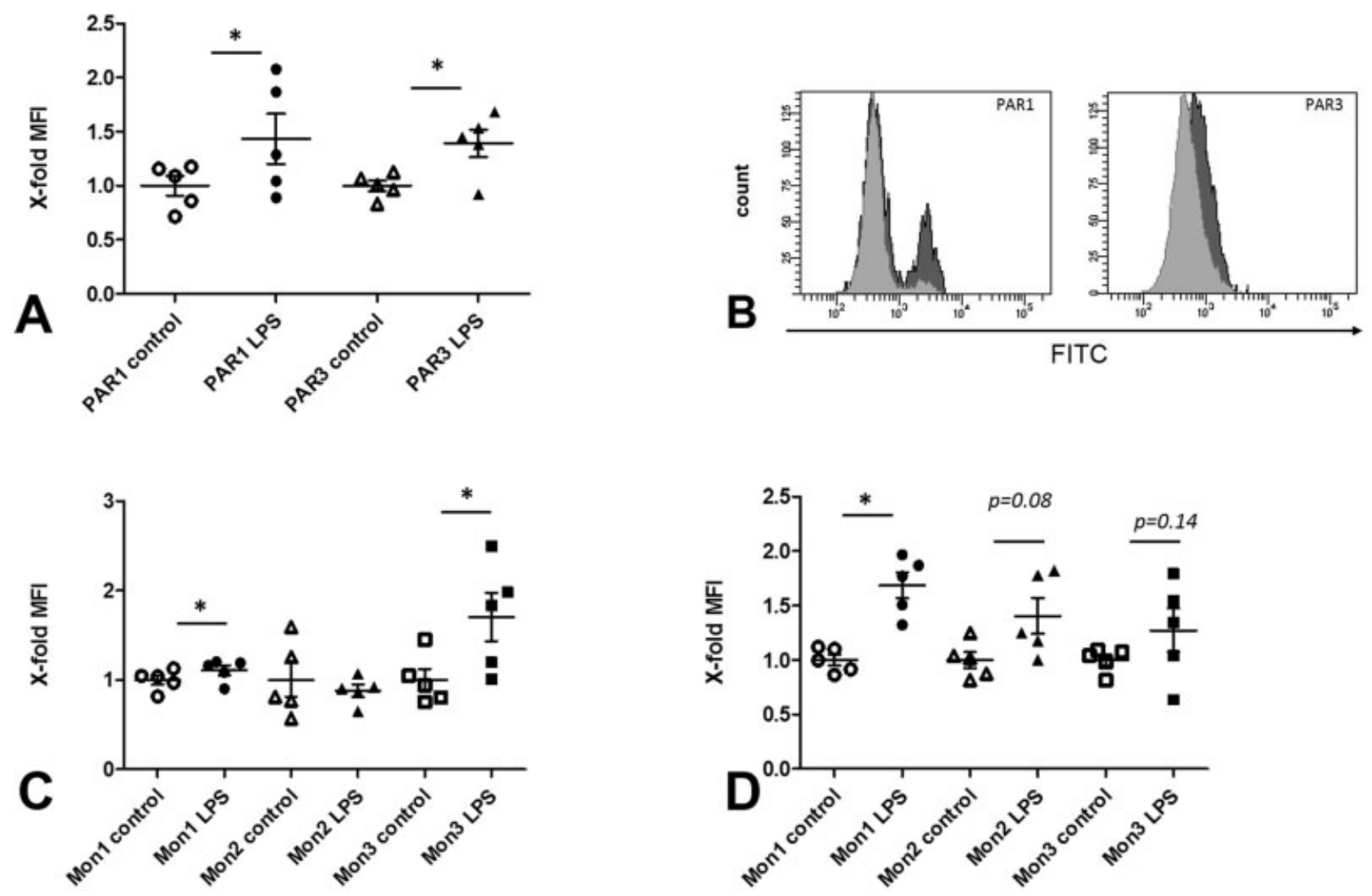

Fig. 3 Induction of protease-activated receptor (PAR) 1 and PAR3 on monocyte subsets by lipopolysaccharide (LPS) in vivo. (A) Flow cytometric analysis of protein levels of PAR1 and PAR3 on total monocytes from whole blood from apparently healthy volunteers that received a bolus of 2 $\mathrm{ng} / \mathrm{kg}$ LPS $(n=5)$. (B) Representative histograms of monocytes stained for PAR1 and PAR3 obtained from one donor before or 1 hour after LPS bolus. (C and D) PAR1 and PAR3 protein levels, respectively, as determined by flow cytometry on monocyte subsets from these blood samples before and 1 hour after LPS infusion $(n=5)$. Values are displayed as $x$-fold mean fluorescent intensities (MFIs) versus unstimulated control \pm standard deviation (SD); ${ }^{*} p<0.05$.

to our knowledge, nothing is known about a possible differential expression of these receptors on human monocyte subsets. Furthermore, information on regulation of the expression of PARs on human monocytes is scarce. ${ }^{7}$ As PAR2 is only activated by unphysiologically high concentrations of thrombin, we focused in this study on the expression of PAR1 and PAR3 and could show, that both receptors are expressed in significantly higher levels on $\mathrm{CD}_{16} 6^{+}$Mon2 and Mon3 as compared with CD16- Mon1. ${ }^{4}$

Proinflammatory cytokines such as IL-1, IL-6, and tumor necrosis factor- $\alpha$ or LPS activate monocytes under inflammatory conditions. ${ }^{1,30}$ Here, we present evidence that LPS added to human whole blood ex vivo upregulates the expression of PAR1 and PAR3 on total human monocytes on the protein level as well as on the level of specific mRNA. It should be noted that brefeldin $\mathrm{A}$, which blocks the protein transport from the endoplasmic reticulum to the Golgi apparatus, did not affect the LPS-induced upregulation of PAR1 and PAR3 in these cells indicating that the majority of the receptor is already available within the cell for transport to the cell surface. These results indicate that these PARs are recruited to the cell surface from a storage pool upon activation with LPS and replenished by mRNA induced-protein synthesis.

When the effect of LPS on subset-specific PAR1 and PAR3 expression was analyzed, we could show that PAR1 was upregulated significantly by LPS on all three subsets of human monocytes ex vivo, whereas a significant increase in PAR3 was only seen in Mon1 and Mon3 under these conditions.

To examine if such an effect of LPS on the expression of PAR1 and PAR3 is also operative in vivo, we analyzed blood samples obtained from healthy volunteers exposed to mild endotoxemia. Endotoxemia significantly upregulated PARs on total monocytes within 1 hour. However, in contrast to our ex vivo findings PAR1 was upregulated significantly only in Mon1 and in Mon3 but not in Mon2, whereas PAR3 was significantly upregulated after 1 hour of endotoxemia in Mon1 with a trend toward significance seen in Mon2 and Mon3. It should be emphasized, however, that our findings were obtained in healthy volunteers exposed to a rather mild and short inflammatory stimulus and thus our results may not resemble the clinical situation in patients suffering from diseases characterized by often long-time chronic inflammation such as coronary artery disease, obesity, or arthritis. In several clinical studies an association of $\mathrm{CD}^{2} 6^{+}$subsets with such pathologies has been described. ${ }^{10-12}$ Our results presented here suggest that mostly the $\mathrm{CD} 16^{-}$Mon1 subset responds to inflammatory activation with an upregulation of PAR1 and PAR3. It would therefore be of interest to determine in future studies if also in chronic inflammatory 

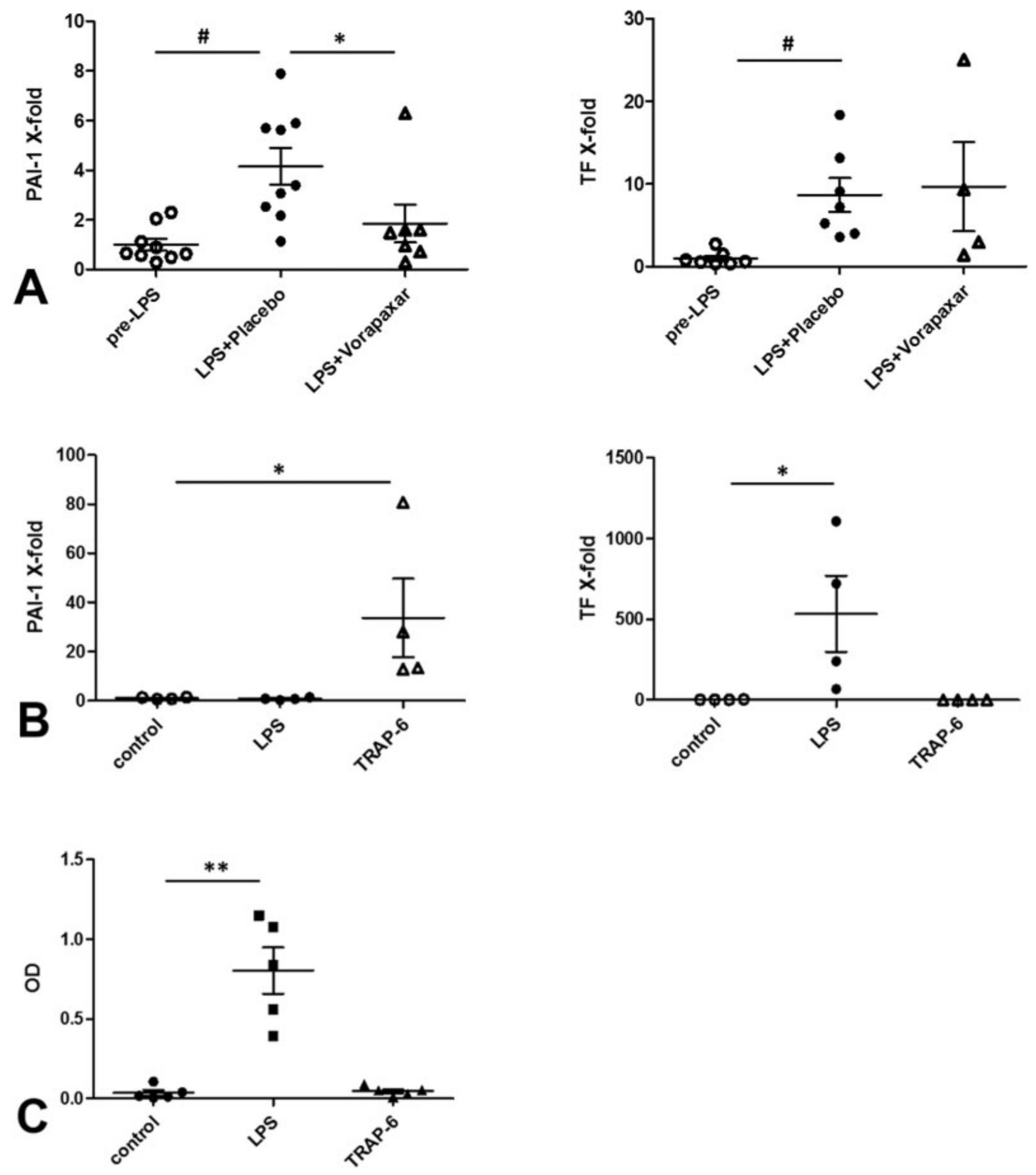

Fig. 4 In vivo and in vitro regulation of plasminogen activator inhibitor-1 (PAI-1) and tissue factor (TF) through PAR1. (A) Messenger ribonucleic acid (mRNA) levels specific for PAl-1 and TF that were determined by real-time polymerase chain reaction (PCR) as described under the "Materials and Methods" section in total monocytes from whole blood from apparently healthy volunteers that received a bolus of $2 \mathrm{ng} / \mathrm{kg}$ lipopolysaccharide (LPS) ( $n=9$ for PAl-1; $n=7$ for TF). (B) mRNA levels specific for PAI- 1 and TF that were determined by real-time PCR as described under the "Materials and Methods" section in total monocytes from whole blood from apparently healthy volunteers that was treated for 4 hours at $37^{\circ} \mathrm{C}$ either with $1 \mu \mathrm{g} / \mathrm{mL}$ LPS, or with $100 \mu \mathrm{M}$ thrombin receptor-activating peptide (TRAP)-6 or left untreated $(n=4)$. (C) TF activity determined as described under the "Materials and Methods" section on microvesicles shed by total monocytes from whole blood from apparently healthy volunteers that was treated for 4 hours at $37^{\circ} \mathrm{C}$ either with $1 \mu \mathrm{g} / \mathrm{mL}$ LPS, or with $100 \mu \mathrm{M}$ TRAP- 6 or left untreated $(n=5)$. Values are displayed as $x$-fold versus unstimulated control \pm standard deviation (SD) (A and B) or optical density (OD) at $405 \mathrm{~nm} \pm \mathrm{SD}(\mathrm{C}) ;{ }^{*} p<0.05,{ }^{* *} p<0.01, \# p<0.0001$. 
pathologies PAR1 and PAR3 are mainly upregulated on Mon1 making them a more sensitized target for activation by thrombin than Mon2 and Mon3.

To investigate whether our finding, that the expression of PARs on monocytes is affected by LPS, is also of relevance in the in vivo situation and might impact on downstream expression of target genes, we interrogated the regulation of two proteins that are regulated by thrombin and are critically involved in thrombus formation and stability, namely, PAI-1 and TF, in the human endotoxemia model. $^{22,23}$ In that respect, it should be noted that, in contrast to TF, PAI-1 is not upregulated by LPS in monocytes. $^{24-28}$ PAI-1 as well as TF expression was significantly upregulated after LPS treatment in the volunteers who were pretreated with placebo. In the group of volunteers who received pretreatment with vorapaxar, a PAR1 antagonist, that has been approved for use as an antiplatelet therapy in treating acute coronary syndromes in the United States, ${ }^{31}$ PAI-1 expression in total monocytes was significantly lower compared with the group of volunteers who received placebo prior to LPS. In contrast, vorapaxar did not change the TF upregulation during endotoxemia. We therefore conclude that LPS-induced TF production in human monocytes in vivo is not dependent on PAR1 as it was not affected by the PAR1 antagonist vorapaxar. This notion is supported by our data showing that the PAR1-agonist TRAP-6 did not affect TF in total monocytes in vitro. The LPS-induced production of PAI-1 in human monocytes in vivo seems to be an indirect effect, which significantly depends on PAR1 because it was almost completely blocked by vorapaxar in vivo, and because it was increased by TRAP- 6 but not by LPS in vitro. One could speculate that in the case of PAI-1, LPS by upregulating PARs on the monocytes renders these cells more susceptible to thrombin formed in this in vivo endotoxemia model. This would be in line with our recent observation that LPS increased prothrombin fragments $\mathrm{F} 1+2$ and thrombin-antithrombin complexes under mild endotoxemia and that this increase was significantly reduced by vorapaxar. ${ }^{21}$

In conclusion, we show here for the first time that PAR1 and PAR3 are differentially expressed on human monocyte subsets with the $\mathrm{CD} 16^{+}$subsets expressing significantly higher levels of both receptors. We also demonstrate that under inflammatory conditions ex vivo and in vivo these receptors are specifically upregulated on particular human monocyte subsets. Furthermore, PAR1 activation seems to be involved in the upregulation of PAI-1 under such LPSmediated inflammatory conditions whereas upregulation of TF occurred independent of PAR1. Some limitations of our study, however, have to be acknowledged: Some of our in vivo data were obtained with unselected, total monocytes only due to limited sample volume resulting in low cell numbers for analysis. On the other hand, when selected monocyte subsets were studied in vitro, effects of culture conditions on subset distribution or reactivity cannot be ruled out completely despite the fact that in vitro culturing was only done for short time periods. Still, our study provides more evidence for a critical role for monocytes as cellular mediators that contribute to the activation of coagulation in diseases characterized by an inflammatory state.

\section{What is known about this topic?}

- Monocytes express protease-activated receptors 1 and 3.

- PARs link inflammation and coagulation.

\section{What does this paper add?}

- $\mathrm{CD}_{16}{ }^{+}$monocytes express more PAR1 and PAR3 than CD16 ${ }^{-}$monocytes.

- PAR 1 and 3 are upregulated by CD16 ${ }^{-}$monocytes in inflammatory conditions in vivo.

- PAR1 activation contributes to a procoagulant milieu through the induction of PAI-1 in monocytes.

Funding

This project was funded by a grant of the Austrian Science Fund FWF (SFB-54) to J.W. and W.S.S.

Conflict of Interest

None declared.

\section{References}

1 Akdis M, Aab A, Altunbulakli C, et al. Interleukins (from IL-1 to IL38 ), interferons, transforming growth factor $\beta$, and TNF- $\alpha$ : Receptors, functions, and roles in diseases. J Allergy Clin Immunol 2016; 138(04):984-1010

2 Borissoff JI, Spronk HMH, Heeneman S, ten Cate H. Is thrombin a key player in the 'coagulation-atherogenesis' maze? Cardiovasc Res 2009;82(03):392-403

3 Hollenberg MD, Mihara K, Polley D, et al. Biased signalling and proteinase-activated receptors (PARs): targeting inflammatory disease. Br J Pharmacol 2014;171(05):1180-1194

4 Isermann B. Homeostatic effects of coagulation protease-dependent signaling and protease activated receptors. J Thromb Haemost 2017;15(07):1273-1284

5 Gieseler F, Ungefroren H, Settmacher U, Hollenberg MD, Kaufmann R. Proteinase-activated receptors (PARs) - focus on receptor-receptor-interactions and their physiological and pathophysiological impact. Cell Commun Signal 2013;11:86

6 Nieman MT. Protease-activated receptors in hemostasis. Blood 2016;128(02):169-177

7 Colognato R, Slupsky JR, Jendrach M, Burysek L, Syrovets T, Simmet T. Differential expression and regulation of protease-activated receptors in human peripheral monocytes and monocyte-derived antigen-presenting cells. Blood 2003;102(07):2645-2652

8 Ziegler-Heitbrock L, Ancuta P, Crowe S, et al. Nomenclature of monocytes and dendritic cells in blood. Blood 2010;116(16):e74-e80

9 Weber C, Shantsila E, Hristov M, et al. Role and analysis of monocyte subsets in cardiovascular disease. Joint consensus document of the European Society of Cardiology (ESC) Working Groups "Atherosclerosis \& Vascular Biology" and "Thrombosis". Thromb Haemost 2016;116(04):626-637

10 Hristov M, Heine GH. Monocyte subsets in atherosclerosis. Hamostaseologie 2015;35(02):105-112

11 Poitou C, Dalmas E, Renovato M, et al. CD14dimCD16+ and CD14+CD16+ monocytes in obesity and during weight loss: 
1402 PARs 1 and 3 and Human Monocyte Subsets Thaler et al.

relationships with fat mass and subclinical atherosclerosis. Arterioscler Thromb Vasc Biol 2011;31(10):2322-2330

12 Weldon AJ, Moldovan I, Cabling MG, et al. Surface APRIL is elevated on myeloid cells and is associated with disease activity in patients with rheumatoid arthritis. J Rheumatol 2015;42(05): 749-759

13 Grip O, Bredberg A, Lindgren S, Henriksson G. Increased subpopulations of CD16(+) and CD56(+) blood monocytes in patients with active Crohn's disease. Inflamm Bowel Dis 2007;13(05): 566-572

14 Mukherjee R, Kanti Barman P, Kumar Thatoi P, Tripathy R, Kumar Das B, Ravindran B. Non-classical monocytes display inflammatory features: validation in sepsis and systemic lupus erythematous. Sci Rep 2015;5:13886

15 Krychtiuk KA, Lenz M, Koller L, et al. Monocyte subset distribution is associated with mortality in critically ill patients. Thromb Haemost 2016;116(05):949-957

16 Thaler B, Hohensinner PJ, Krychtiuk KA, et al. Differential in vivo activation of monocyte subsets during low-grade inflammation through experimental endotoxemia in humans. Sci Rep 2016;6:30162

17 Jilma-Stohlawetz P, Kliegel T, Kantner-Schlifke I, Strasser-Marsik C, Mayr FB, Jilma B. Upregulation of cytokine mRNA in circulating leukocytes during human endotoxemia. Eur Cytokine Netw 2017; 28(01):19-26

18 Mayr FB, Spiel AO, Leitner JM, et al. Duffy antigen modifies the chemokine response in human endotoxemia. Crit Care Med 2008; 36(01):159-165

19 Stojkovic S, Thulin $\AA$, Hell L, et al. IL-33 stimulates the release of procoagulant microvesicles from human monocytes and differentially increases tissue factor in human monocyte subsets. Thromb Haemost 2017;117(07):1379-1390

20 Khorana AA, Francis CW, Menzies KE, et al. Plasma tissue factor may be predictive of venous thromboembolism in pancreatic cancer. J Thromb Haemost 2008;6(11):1983-1985
21 Schoergenhofer C, Schwameis M, Gelbenegger G, et al. Inhibition of protease-activated receptor (PAR1) reduces activation of the endothelium, coagulation, fibrinolysis and inflammation during human endotoxemia. Thromb Haemost 2018;118(07):1176-1184

22 Grover SP, Mackman N. Tissue factor: an essential mediator of hemostasis and trigger of thrombosis. Arterioscler Thromb Vasc Biol 2018;38(04):709-725

23 Jung RG, Simard T, Labinaz A, et al. Role of plasminogen activator inhibitor-1 in coronary pathophysiology. Thromb Res 2018; 164:54-62

24 Hamilton JA, Whitty GA, Wojta J, Gallichio M, McGrath K, Ianches G. Regulation of plasminogen activator inhibitor-1 levels in human monocytes. Cell Immunol 1993;152(01):7-17

25 Mackman N. Regulation of the tissue factor gene. FASEB J 1995;9 (10):883-889

26 Derhaschnig U, Bergmair D, Marsik C, Schlifke I, Wijdenes J, Jilma B. Effect of interleukin-6 blockade on tissue factor-induced coagulation in human endotoxemia. Crit Care Med 2004;32(05):1136-1140

27 Hamilton JA, Whitty GA, Stanton H, et al. Macrophage colonystimulating factor and granulocyte-macrophage colony-stimulating factor stimulate the synthesis of plasminogen-activator inhibitors by human monocytes. Blood 1993;82(12):3616-3621

28 Gregory SA, Morrissey JH, Edgington TS. Regulation of tissue factor gene expression in the monocyte procoagulant response to endotoxin. Mol Cell Biol 1989;9(06):2752-2755

29 Posma JJN, Posthuma JJ, Spronk HMH. Coagulation and noncoagulation effects of thrombin. J Thromb Haemost 2016;14 (10):1908-1916

30 Egorina EM, Sovershaev MA, Hansen J-B. The role of tissue factor in systemic inflammatory response syndrome. Blood Coagul Fibrinolysis 2011;22(06):451-456

31 Tricoci P, Huang Z, Held C, et al; TRACER Investigators. Thrombinreceptor antagonist vorapaxar in acute coronary syndromes. N Engl J Med 2012;366(01):20-33 